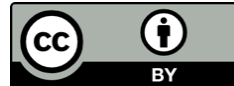

\title{
Impacto de la reforma del impuesto a la renta MYPE para el crecimiento de la micro y pequeña empresa en el distrito de Los Olivos 2017
}

Impact of the MYPE income tax reform for the growth of micro and small businesses in the district of Los Olivos 2017 Impacto da reforma do imposto de renda MYPE para o crescimento das micro e pequenas empresas no distrito de Los Olivos 2017

\section{ARTÍCULO GENERAL}

\author{
Williams Arturo Martínez Aberga \\ https://orcid.org/0000-0002-1608-6895 \\ will-mart@hotmail.com \\ Universidad Católica Sedes Sapientiae, Lima-Perú
}

Recibido 14 de Diciembre 2020 | Arbitrado y aceptado 06 de Enero 2020 | Publicado el 01 de Marzo 2021

\section{RESUMEN}

Objetivo: El objetivo principal del presente estudio consiste en determinar el impacto del RMT en el crecimiento de las micro y pequeñas empresas de los sectores, comercio, industria y servicios del distrito de Los Olivos. Método: Para lograr este objetivo se ha empleado información de 12 empresas categorizadas como micros y pequeña empresa del distrito de Los Olivos. Corresponde a una investigación no experimental, transversal, la investigación se realizó con información del año 2017, y es de nivel correlacional porque se busca encontrar la relación causa y efecto entre las variables de estudio. Resultados: Se presentan en relación al sector económico donde se desarrolla la MYPE y también sobre la base de indicadores de pago de impuestos, utilidades, activos, y empleo. Conclusión: La principal conclusión que arroja el estudio es que el RMT, sí ejerció un impacto medianamente significativo sobre el crecimiento empresarial de las MYPE de Los Olivos.

PALABRAS CLAVE: Reforma; impuesto; MYPE; formalización; ingresos; crecimiento.

\section{ABSTRACT}

Objective: The main objective of this study is to determine the impact of the RMT on the growth of micro and small companies in the sectors, commerce, industry and services in the district of Los Olivos. Method: To achieve this objective, information from 12 companies categorized as micro and small companies in the district of Los Olivos has been used. It corresponds to a non-experimental, cross-sectional investigation, the investigation was carried out with information from the year 2017, and it is of a correlational level because it seeks to find the cause and effect relationship between the study variables. Results: They are presented in relation to the economic sector where the MYPE is developed and also based on indicators of tax payment, profits, assets, and employment. Conclusion: The main conclusion of the study is that the RMT did have a moderately significant impact on the business growth of MYPEs in Los Olivos.

KEY WORDS: Reform; tax; MYPE; formalization; income; growth.

\section{RESUMO}

Objetivo: O principal objetivo deste estudo é determinar o impacto do RMT no crescimento das micro e pequenas empresas dos setores, comércio, indústria e serviços no distrito de Los Olivos. Método: Para atingir este objetivo, foram utilizadas informações de 12 empresas categorizadas como micro e pequenas empresas do distrito de Los Olivos. Corresponde a uma investigação não experimental, transversal, a investigação foi realizada com informação do ano de 2017, e é de nível correlacional porque procura encontrar a relação de causa e efeito entre as variáveis do estudo. Resultados: São apresentados em relação ao setor econômico onde o MYPE é desenvolvido e também com base em indicadores de pagamento de impostos, lucros, patrimônio e emprego. Conclusão: A principal conclusão do estudo é que o RMT teve um impacto moderadamente significativo no crescimento dos negócios dos MYPEs em Los Olivos.

PALAVRAS-CHAVE: Reforma; imposto; MEU PE; formalização; renda; crescimento. 


\section{Introducción}

El Estado como parte de su política tributaria, ha asumido el importante rol de fomento de la inclusión de las MYPE a través de distintas reformas tributarias implementadas en los últimos años, para que puedan contribuir en el incremento de la recaudación fiscal, otorgando facilidades de acceso a la formalidad lo que reduce costos y proporciona beneficios. La formalidad en un micro y pequeño empresario y la mejora de su competitividad, pueden conseguir un efecto satisfactorio en la sociedad, no solo porque se incrementaría la base tributaria del país y en consecuencia los ingresos fiscales, sino porque se lograría una mejora de la calidad de vida de aquellos emprendedores que con mucho esfuerzo tratan de impulsar sus negocios, en bien de sus familias lo que repercute en el bienestar general.

Con respecto al RMT, a fines de diciembre del 2016, el Ejecutivo publicó el decreto legislativo $N^{\circ} 1269$ que crea el Régimen MYPE Tributario del Impuesto a la Renta, que comprende a las micro y pequeñas empresas que tengan como requisito que sus ingresos netos no superen las 1,700 Unidades Impositivas Tributarias (UIT) en el ejercicio gravable. Ello con el objetivo de ampliar la base tributaria dando facilidad a un sector de gran participación en la economía del país, pero con bajo nivel de formalización tributaria.

En este contexto, países como el Perú que tratan de mejorar las condiciones de vida de su población, las empresas cumplen un rol fundamental, ya sea produciendo bienes, servicios, sea como compañías comerciales, industriales, extractivas, y de servicios, contribuyendo de manera importante en la actividad económica y la generación de empleo. Por lo que se debe incentivar a través de la política tributaria, el crecimiento de la MYPE

La política fiscal moderna reconoce que los tributos cumplen una doble función: el de recaudar recursos para que el Estado satisfaga las necesidades de la población, con énfasis en los más necesitados, y la otra como instrumento de política social y económica con el propósito de poder financiar la puesta en marcha de programas de interés social. En este sentido, según la Carta Magna del Perú 1993, el estado tiene la potestad de implementar en nuestro país un sistema tributario mediante la cual puede crear, modificar los tributos que sean suficientes con la finalidad de coadyuvar en el crecimiento de las empresas. 
En este sentido, cabe señalar que según la Sociedad de Comercio Exterior del Perú (Comex), las cifras del 2016, en el Perú 9 de cada 10 empresas son micro y pequeñas (MYPE) las que aportan del PBI un 21,6\%, sin embargo el 83,1\% del total de estas compañías son informales. En estas empresas trabajan 7,7 millones de personas, de esta cantidad el 66,5\% no perciben sueldo ni seguro de salud o sistema previsional, ello debido a que el $72,5 \%$ constituye mano de obra familiar.

Las micro y pequeñas empresas en el país sumaban 5,7 millones en el 2016, pero la informalidad limita su crecimiento. En Perú, los negocios que pertenecen a la división de las MYPE son un 96.5\%, cantidad que va en aumento año tras otro, mientras que el número de medianas y grandes empresas se redujo comparado al año anterior. Sin embargo, se señala que cerca del $80 \%$ de estas aún son informales y que en un $65.3 \%$ corresponden a empresas familiares. Por lo que es necesario que la MYPE sea eficiente y productiva para seguir impulsando su crecimiento, porque es importante que puedan ganar participación en el mercado.

Esta falta de competitividad se refleja en que el 56\% microempresas realizan exportaciones, aunque esta cifra ha disminuido en 1,5\% en el 2017 en relación al periodo 2016. Los envíos de las MYPE al exterior significan solo un 2,2\% de la cifra final de las exportaciones nacionales, mientras que las entregas de las empresas medianas y grandes constituyen el 97,8\% de las exportaciones. Lo que refleja la problemática que se observa en las MYPE.

Según información proporcionada en el año 2016 por la Administración Tributaria, las microempresas representan el 95.6\% de las MYPE pero sólo aportan el 36.7\% del total de la recaudación. La cantidad de pequeñas empresas representan sólo el $4.4 \%$ pero contribuyen con una recaudación del $63.3 \%$. Esta es la explicación de porqué de los 8.2 millones de empresas que se encontraban registrados, solo 1.76 millones de micro y pequeñas empresas informan sobre sus actividades que generan impuestos por rentas de tercera categoría, pero solo 1.3 millones contribuyentes paga mencionado impuesto. Como se observa en estas cifras, el objetivo de ampliar la base, para tener mayor recaudación, ello no está ocurriendo ya que, los que estaban en el Régimen General, que eran los que pagaban más impuestos, están cambiando a un régimen en la que van a contribuir con menos tributos. 
Las ventas de las micro y pequeñas empresas se han venido reduciendo en los últimos años. Según información tomada de la página de COMEX, el nivel de ventas de las MYPE se redujo en el 2017, ya que de acuerdo a la Encuesta Nacional de Hogares (ENAHO), las ventas para el 2017 alcanzaron un total de S/ 135,000 millones, lo que representó un $0.7 \%$ menos que lo registrado en 2016 . Ello a pesar que el número de micro y pequeñas empresas que existe en el país ascendió a 5.9 millones en 2017, lo que significó un aumento del 3.8\% con respecto al año 2016, sin embargo, su nivel de ventas cayó.

Se ha incrementado, en los últimos años, la cantidad de empresas que se registraron en regímenes tributarios simplificados porque ofrecen menor pago de impuestos. Se indica que, si en el año 2015 las empresas que pertenecía a algún régimen tributario especial representaban el 68\%, este porcentaje aumentó en el 2016 hasta el 70\%. No obstante, al crearse el Régimen Mype Tributario (RMT) en el 2016, el número de compañías inscritas a un régimen simplificado se elevó en el 2017 hasta el 89\%.

Según cifras de la Administración Tributaria, de enero a julio del 2017 se cobró S/ 406 millones 4 mil de impuestos a través del (RMT), monto que aumento en un 78,4\% para el mismo periodo del año 2018 en que se recaudó S/ 731 millones 2 mil, lo cual muestra el aumento de empresas que se registran en este sistema para contribuir con menos impuestos. Por lo que se requiere realizar ajustes para que en los casos donde exista una presunción de división empresarial, se evite que se incluyan compañías que no deberían gozar de este beneficio.

En el Régimen MYPE Tributario (RMT), en comparación al Régimen Especial de Renta (RER), que se determina con el $1.5 \%$ de los ingresos mensuales como pago definitivo de impuesto a la renta, en el nuevo régimen se determina el impuesto sobre las utilidades netas, lo que consta de dos niveles: las compañías que obtengan ganancias hasta a 15 UIT (S/ 60,750) calculan el impuesto con una tasa de 10\%, mientras que las demás empresas que tienen ganancias por más de 15 UIT, deben calcularlo con una tasa de $29.5 \%$, esto significa entonces tener en la práctica la misma tasa que las empresas grandes. De esta forma, desde la inclusión del RMT, se cuenta entonces con tres regímenes orientados a la MYPE: RUS, RER y el RMT. Entonces, una compañía que obtiene ingresos máximos de S/ 96,000 en el año, puede acogerse a cualquiera de los 
mencionados regímenes. Mientras, que las empresas que obtienen ingresos entre $\mathrm{S} /$ 96,000 y S/ 525,000 en el año pueden ubicarse en el RER o el RMT; mientras que aquellas que obtengan ingresos por más de S/ 525,000 anuales solo podrán estar en el RMT.

De esta forma, el estudio resulta importante de realizar porque con esta investigación se puede conocer en mayor medida la relación que existe entre las variables en estudio, además que sirve de gran ayuda para nuevas investigaciones y teorías que se planteen en el campo de las medidas que se pueden dictar para que se implemente una política tributaria que tenga un impacto positivo sobre el crecimiento empresarial de la MYPE, de gran relevancia en la economía del país, por su contribución a la producción y el empleo.

Se plantea como hipótesis principal del presente estudio, que la Reforma del Impuesto a la Renta ha tenido un impacto medianamente significativo en el crecimiento de las micro y pequeñas empresas del distrito de Los Olivos, y su efecto ha sido de distinta magnitud en los sectores, comercio, industria y servicios en el periodo 2017

En lo referido al sustento teórico del presente estudio, autores como Bird y Wilkie (2012), señalan que:

La política tributaria es fundamental para un país, ya que los impuestos son necesarios tanto para financiar el gasto público deseado como para garantizar que la carga a pagar por dicho gasto se distribuya de una manera que sea administrativamente viable, económicamente sostenible y políticamente aceptable. Los ciudadanos a través de sus instituciones políticas pueden elegir consumir colectivamente de la misma manera que los hogares asignan el presupuesto familiar. Por supuesto, al igual que en una familia, no todos son generadores de ingresos, así que podemos elegir compartir - redistribuir - buena parte de nuestros ingresos colectivos para asegurar que aquellos con ingresos menores o nulos no sean excluidos de bienes públicos como educación o salud o para complementar su capacidad de obtener bienes de provisión privada como comida o refugio, que resultan importantes para su bienestar y calidad de vida. Los impuestos constituyen así, el precio que pagamos por la civilización. El consumo colectivo efectuado a través de impuestos facilita la sociedad civil y establece sus límites. Las oportunidades privadas de beneficio y ganancia dependen en gran medida de la existencia de una sociedad civil que permite y 
alienta a las personas a participar, de manera segura, en una variedad de relaciones sociales, políticas y económicas. (p.2)

De acuerdo a Norregaard \& Khan (2007), con respecto a la política tributaria, señalan que:

Un tema central de política en los últimos años ha sido las implicaciones para la estabilidad de las bases tributarias de la integración económica y la movilidad cada vez mayor del capital (tanto físico como financiero), laboral y de bienes y servicios. Los países en desarrollo se centran, en particular, en atraer inversiones y aumentar los ingresos para promover el crecimiento, y los países desarrollados se preocupan principalmente por salvaguardar sus bases fiscales para preservar el estado del bienestar y para hacer frente a los retos del envejecimiento de su población. (p.3)

Macek (2014) explica que:

La sociedad globalizada actual se caracteriza por la necesidad de la existencia de procesos de redistribución debido a que debe cumplir sus funciones como Estado. Al mismo tiempo, el gasto del gobierno como herramienta básica de la política económica está condicionado por la necesidad de su financiamiento, donde los ingresos fiscales generalmente representan la parte más significativa de los ingresos del presupuesto estatal. Sin embargo, la propia estructura del sistema tributario, la combinación impositiva, las desgravaciones fiscales y las recargas fiscales son objeto de gran debate nacional. Al mismo tiempo, muchos países desarrollados se ven afectados actualmente por la importante crisis presupuestaria en la que tienen problemas para pagar sus obligaciones a corto y largo plazo. Debido a esto, los propios gobiernos están expuestos a una mayor supervisión de los mercados financieros y, por lo tanto, se ven obligados a consolidar los presupuestos públicos. La crisis de las finanzas públicas se resuelve generalmente por dos canales concretos: (i) Por el canal de reducción del gasto público, y (ii) El canal de aumento de impuestos, o ingresos fiscales. Por un lado, el objetivo básico de la consolidación es mantener los criterios de responsabilidad del Presupuesto Público, según lo proyectado, y por otro lado, restaurar el crecimiento económico tan pronto como sea posible. (p.2) 
Jones y Manuelli (2001) por otro lado refieren que: "En el análisis del crecimiento económico, el capital humano es el factor más influenciado por los impuestos. Debido al crecimiento del producto marginal, el capital humano tiene un efecto tal que la inversión en educación es efectiva en economías que se encuentran en estado estable" (p30).

Una relación positiva entre las inversiones en capital humano y el crecimiento económico a largo plazo se confirmó en muchos estudios, como por ejemplo, los de Teixeira y Fortuna (2003).

Jacobs (2007) señala que: "Puede existir una dependencia positiva entre el crecimiento económico y los impuestos si los ingresos de los impuestos se usan solo para la acumulación de capital humano. Las desgravaciones fiscales son el elemento de motivación más importante para que el empleador invierta en capital humano" (p219).

Davidsson (1989) señaló que: "Un sistema impositivo desfavorable, reglas y regulaciones complejas puede llegar a constituir un obstáculo para el crecimiento de las pequeñas empresas" (p20).

Barbarán et al. (2018) en su investigación refiere que:

Las MYPE constituyen un sector económico productivo que contribuyen de manera significativa al crecimiento, bienestar y crecimiento del país, por lo que se debe prever todo aquello que permita su expansión y mejora, ya que ello redunda en el mayor bienestar de la población. Entre los resultados obtenidos en su estudio, indica que el "sistema tributario" fue identificado como el principal limitante para el crecimiento empresarial de las micro y pequeñas empresas de la provincia del Santa, y por tanto, es la dimensión Estado la que mayormente influye con un 47\%, en los problemas de crecimiento del negocio y su competitividad. (p.3)

Asimismo, otras investigaciones como los de Zevallos (2017), indica:

Para el 88\% de los empresarios encuestados, el Régimen MYPE Tributario influye significativamente y de manera positiva en el Desarrollo Empresarial en las Empresas Transporte de Carga del Distrito de Huánuco-2017, permitiéndoles de esta manera mejorar su competitividad empresarial y posicionamiento en el mercado. (p.48) 


\section{Método}

La presente investigación es no experimental, ya que no se manipularon las variables en estudio como son las referidas a la reforma tributaria RMT como variable independiente y el crecimiento empresarial de las MYPE, medido a través de las ventas, activos, utilidades, pago del impuesto a la renta y empleo generado, que efectúan las micro y pequeñas empresas en el distrito de Los Olivos.

Es transversal, porque la investigación se realizó en un solo momento en el tiempo, año 2017, y es de nivel correlacional porque se busca encontrar la relación causa y efecto entre ambas variables.

La población está compuesta por las MYPE del distrito de Los Olivos, mientras que la muestra sigue el criterio de muestreo de casos tipo según indica Hernández et al. (2014, p. 387). Según este texto, la muestra de casos tipo se puede utilizar en estudios cuantitativos de tipo exploratorio en el que el objetivo es la riqueza de la información, la profundidad y calidad de los datos que se obtienen, no la cantidad ni la estandarización.

La presente investigación, analiza el caso de 12 MYPE del distrito de Los Olivos. Se buscó un número homogéneo para el estudio, las MYPE elegidas se dividen en cuatro del sector comercio, cuatro del sector industria y cuatro del sector servicios. Se consideró también para la elección de la muestra, que estas MYPE cuenten con más de 3 años en el mercado, ya que es un periodo mínimo en el cual se puede evaluar sus resultados económicos y financieros y sirvan para el análisis comparativo. Además se tomó en consideración para la muestra solo a las empresas que elaboran sus estados financieros y lo presentan a la Administración Tributaria y se encuentren en el Régimen MYPE tributario.

Estas doce microempresas de sectores económicos diversos como comercio, industria y servicios, constituye una muestra representativa como estudio de caso para la investigación.

Entre los limitantes al desarrollo de la investigación cabe mencionar que dada la gran cantidad de micro y pequeñas empresas en el distrito de Los Olivos, y su elevado nivel de informalidad, lo que significa que no elaboran los estados financieros del negocio, se decidió bajo un criterio discrecional del investigador, tomar una muestra de 
12 MYPE, que fue el número de empresarios que sí estuvieron dispuestos a brindar información financiera de sus empresas.

Se recabo los estados financieros, el estado de resultados y el estado de situación financiera, además se realizó una encuesta a un conjunto de 12 MYPE de los sectores comercio, industria y servicios del distrito de Los Olivos.

El procesamiento de datos se realizó en SPSS, ya que se busca determinar una relación causal entre una variable dependiente y una variable exógena, para lo cual se realizará un análisis regresional.

\section{Resultados}

Para la presente investigación se estableció los siguientes criterios para dar a conocer los resultados, se utilizara la expresión poco significativa o medianamente significativa o bastante significativa. Cuya definición para la investigación es:

Poco significativo, cuando tres indicadores han tenido una disminución porcentual.

Medianamente significativo, cuando como mínimo dos indicadores han tenido un aumento porcentual y estos resultados son superiores a $30 \%$ por cada indicador.

Bastante significativo, cuando tres o más indicadores han tenido un aumento porcentual en sus resultados y estos resultados son mayores a $60 \%$ por cada indicador.

Para el análisis de los resultados, consideramos a las ventas como indicador de crecimiento empresarial, en el sentido que de acuerdo a la definición, el crecimiento económico se refiere a incremento constante de la producción, lo que se refleja en mayores ventas para la empresa. Para ello se obtiene la siguiente información de una muestra de 12 microempresas, acogidas a la reforma tributaria MYPE, lo que se presenta en el siguiente gráfico. 


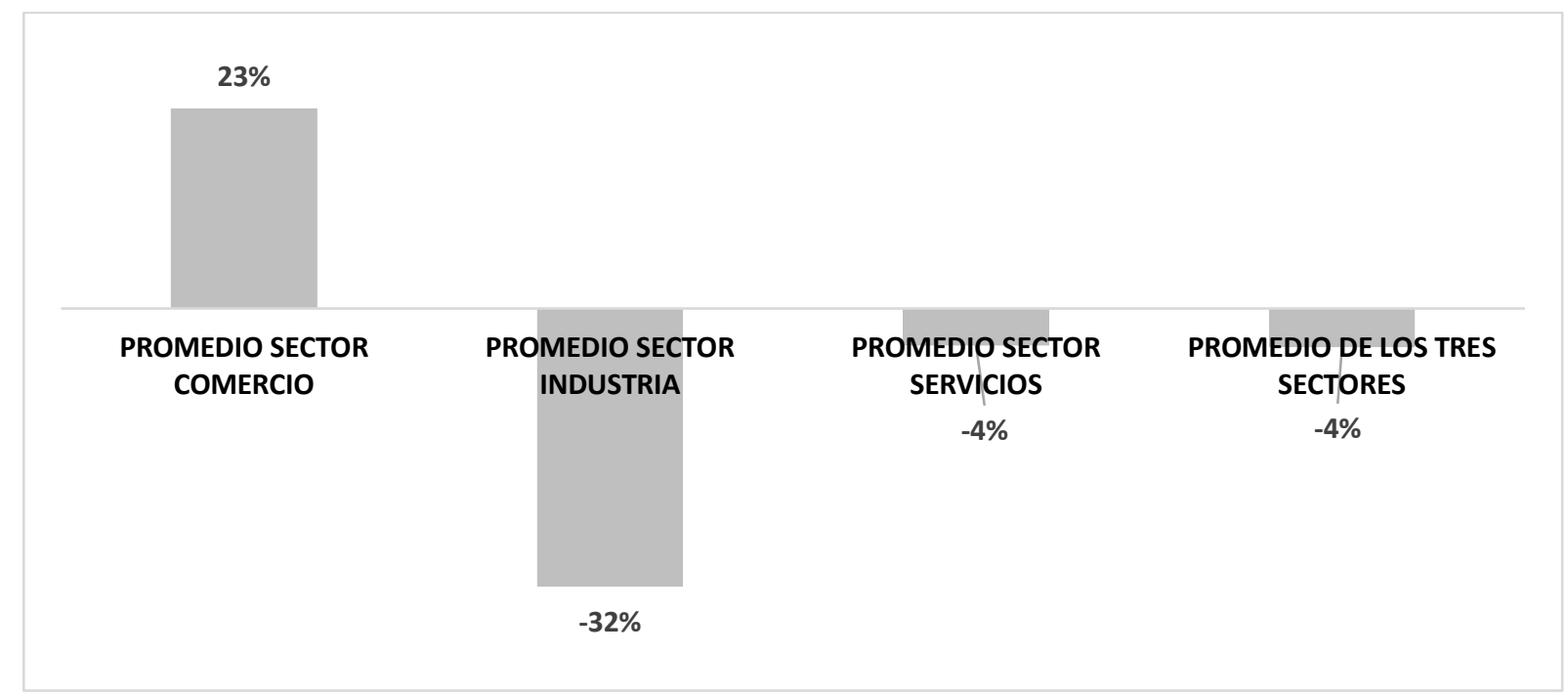

Figura 1: Variación porcentual promedio de las ventas de una muestra de 12 empresas en el distrito de Los Olivos (2016 - 2017)

Fuente: Encuesta realizada a muestra de MYPE en Los Olivos - Elaboración propia

Estos resultados indican que luego de la entrada en vigencia del Régimen MYPE Tributario a partir de enero del año 2017, ha tenido un impacto poco significativo, en la muestra de 12 empresas que se acogieron a dicho régimen, se observa que de manera general las ventas cayeron en un 4\%. A nivel de sectores vemos que el sector que creció fue el de comercio (23\%), mientras que los que decayeron fueron industrial $(32 \%)$, y servicios (4\%). Sin embargo, quizá falte un poco más de tiempo para evaluar el impacto sobre las ventas, ya que si la facilidad tributaria se dirigió a mayor inversión, se requeriría de un mayor plazo para que ello rinda sus frutos en mayores ventas en los sectores industria y servicios.

Visto los resultados por sector económico, se tiene que en el sector comercio el Régimen MYPE Tributario ha tenido un impacto medianamente significativo, si bien cae el pago de impuesto a la renta (-66\%), debido al cambio de alícuota al 10\%, se observa un resultado favorable sobre los activos (sube 35\%) y las ventas (sube 23\%), y de manera significativa sobre las utilidades (incremento de 105\%). 


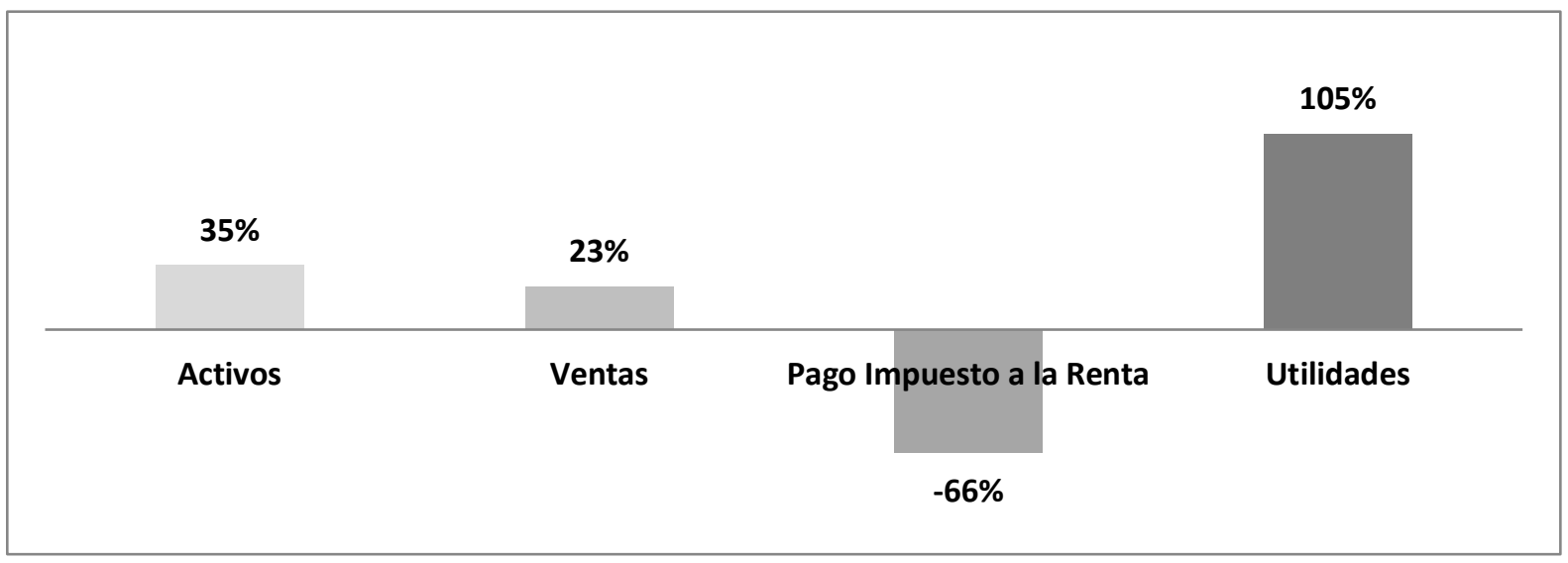

Figura 2: Promedio del sector comercio (Variación porcentual promedio de una muestra de 12 empresas en el distrito de Los Olivos, 2016 - 2017)

Fuente: Encuesta realizada a muestra de MYPE en Los Olivos - Elaboración propia

En el sector industria el Régimen MYPE Tributario ha tenido un impacto poco significativo, cae el pago de impuesto a la renta (-46\%), las ventas (-32\%) y las utilidades $(-82 \%)$, y se observa un resultado favorable sobre los activos (sube $45 \%$ ), lo que muestra que en este sector de manera específica el impacto no ha sido favorable. Sí ha habido un incremento de los activos, lo que refleja capacidad de inversión y de acumular para el largo plazo.

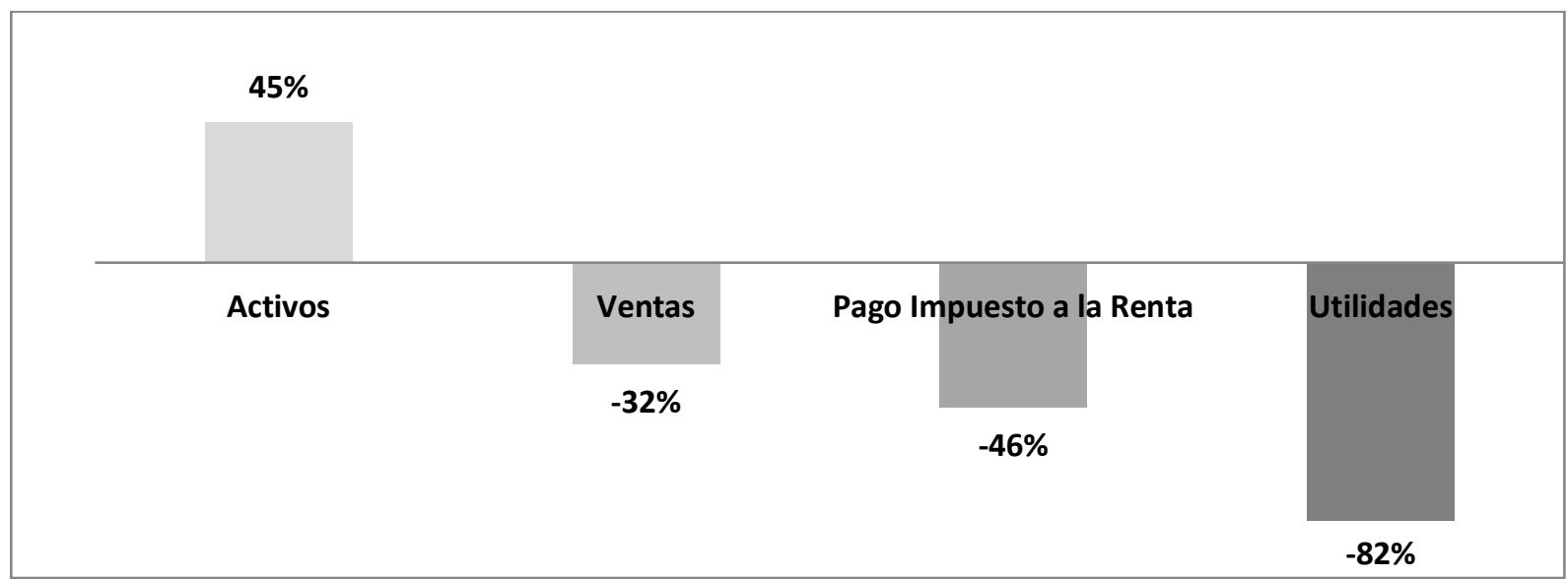

Figura 3: Promedio sector industria (Variación porcentual promedio de una muestra de 12 empresas en el distrito de Los Olivos, 2016 - 2017)

Fuente: Encuesta realizada a muestra de MYPE en Los Olivos - Elaboración propia 
Para el caso del sector servicios, el Régimen MYPE Tributario ha tenido un impacto medianamente significativo, si bien las ventas caen un poco (-4\%), hay un incremento en los activos (9\%), pero sobre todo en las utilidades (159\%) y más aún en el pago del impuesto a la renta (1230\%), lo que muestra que este significativo aumento se da por aquellas empresas que antes del RMT no pagaban este impuesto, y que ahora sí lo hacen al haberse acogido a este régimen.

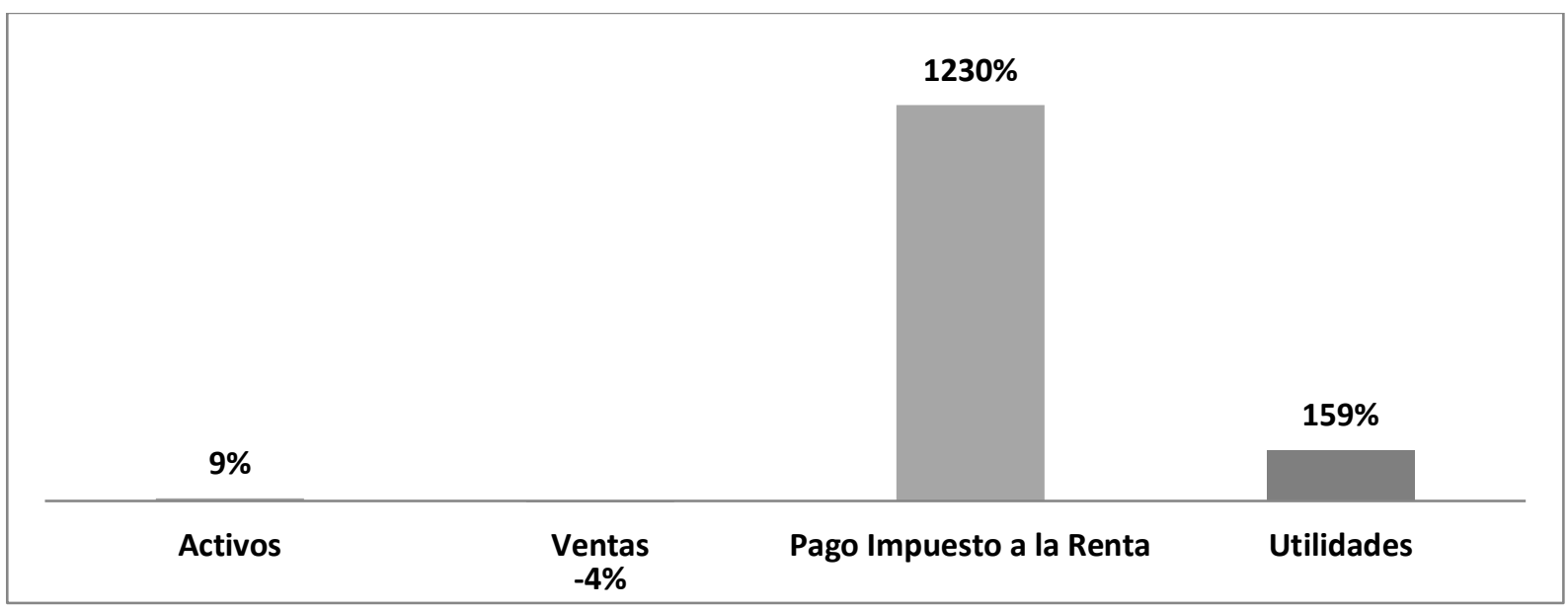

Figura 4: Promedio sector servicios (Variación porcentual promedio de una muestra de 12 empresas en el distrito de Los Olivos, 2016 - 2017)

Fuente: Encuesta realizada a muestra de MYPE en Los Olivos - Elaboración propia

El Régimen MYPE Tributario ha tenido un impacto poco significativo sobre el pago del impuesto a las ganancias de la MYPE de los sectores, comercio, industria y servicios, del distrito de Los Olivos, tal como se observa en el siguiente gráfico.

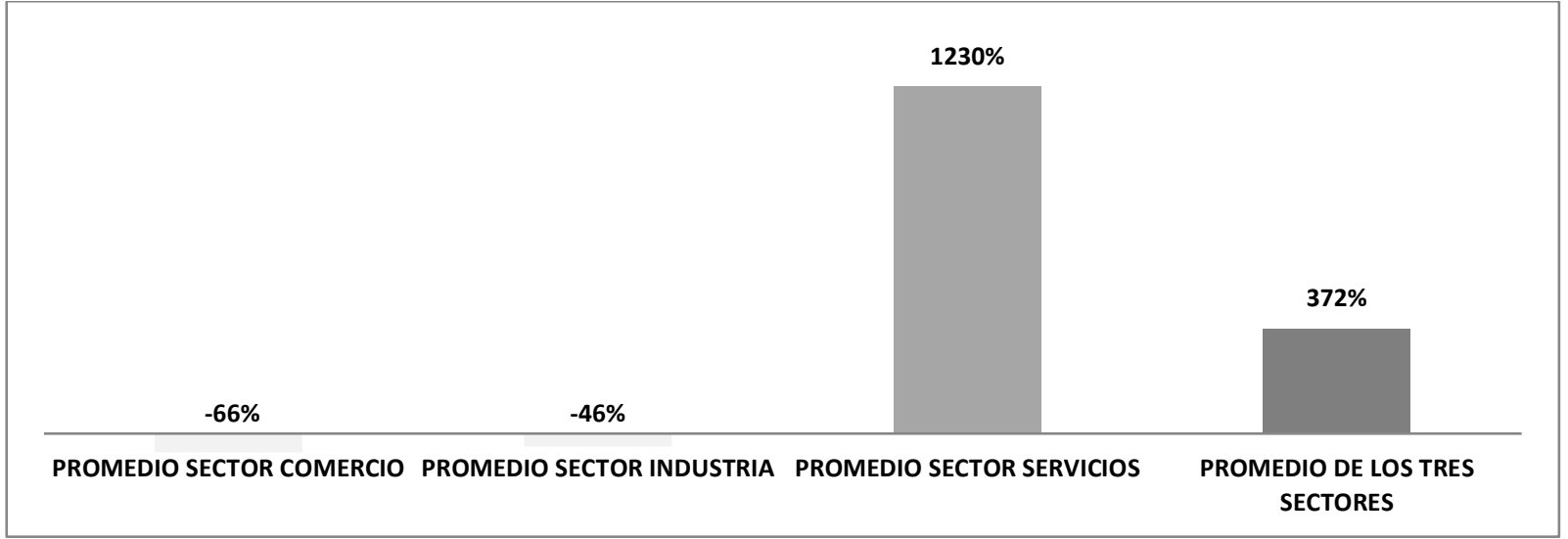


Figura 5: Variación porcentual de pago de Impuesto a la Renta en el distrito de Los Olivos (2016 - 2017)

Fuente: Encuesta realizada a muestra de MYPE en Los Olivos - Elaboración propia

Vemos que si bien los pagos por concepto de impuesto a la renta cayeron a su entrada en vigencia en los sectores comercio (-66\%) e industria (-46\%), en el sector servicios sí se observa un significativo incremento (1230\%), sobre todo de empresas que no pagaban este impuesto, y que lo empezaron a hacer desde enero del 2017, que empieza a aplicarse la alícuota reducida del 10\%. Por lo que de manera global tenemos un incremento de $372 \%$. Tal como se muestra entonces, el impacto del RMT ha sido diferenciado a nivel de los sectores comercio, industria y servicios, lo que refleja que cada sector tiene características distintas.

Otro dato importante es el referido a que el Régimen MYPE Tributario ha tenido un impacto bastante significativo sobre las utilidades de las micro y pequeñas empresas de los sectores, comercio, industria y servicios, del distrito de Los Olivos.

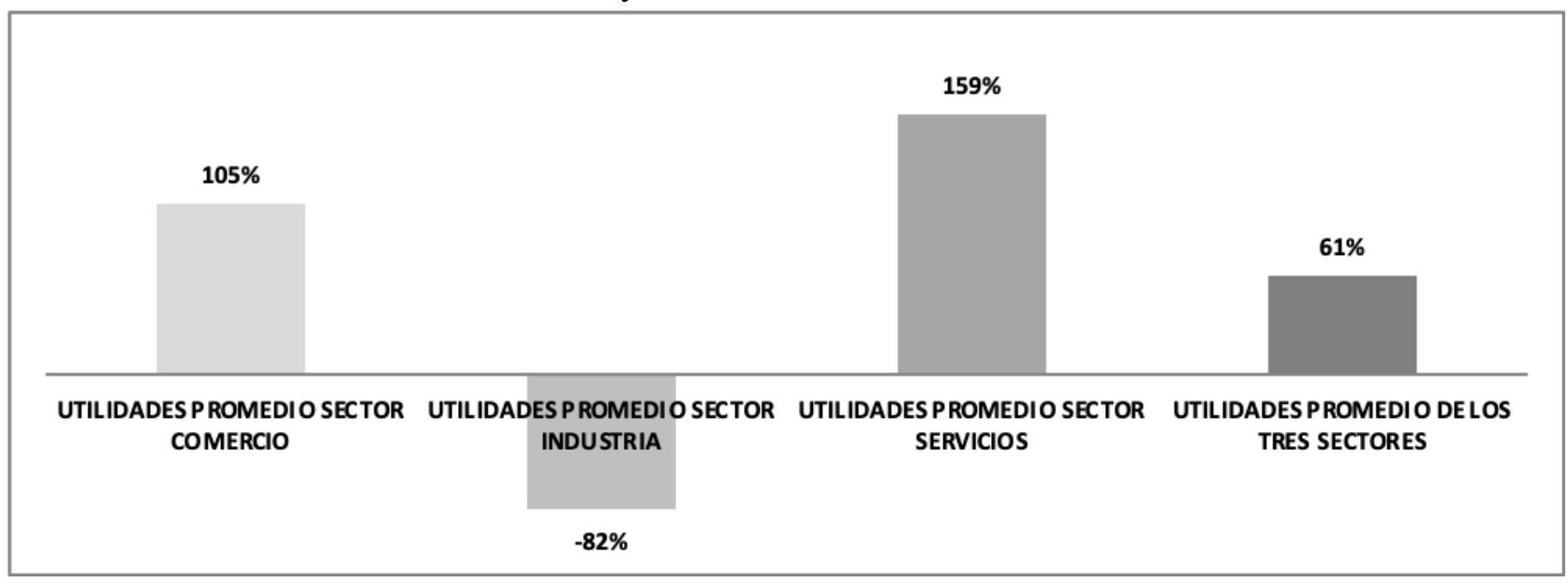

Figura 6: Variación porcentual de las utilidades de una muestra de 12 empresas en el distrito de Los Olivos (2016 - 2017)

Fuente: Encuesta realizada a muestra de MYPE en Los Olivos - Elaboración propia

En este caso se observa que de manera general el impacto del Régimen MYPE Tributario ha sido positivo sobre las utilidades, dado que se observa un incremento en el 2017 , con respecto al 2016, de 61\%, y en el sector comercio se dio un aumento de $105 \%$, 
ocasionado por una mejor gestión de los gastos y servicios recibidos de terceros, salvo de manera específica en el sector industria, donde se observa una caída de $82 \%$, por una mayor inversión en activos y su depreciación, además de una mayor contratación de mano de obra.

Asimismo, se tiene que el Régimen MYPE Tributario ha tenido un impacto medianamente significativo sobre los activos de las micro y pequeñas empresas de los sectores, comercio, industria y servicios, del distrito de Los Olivos.

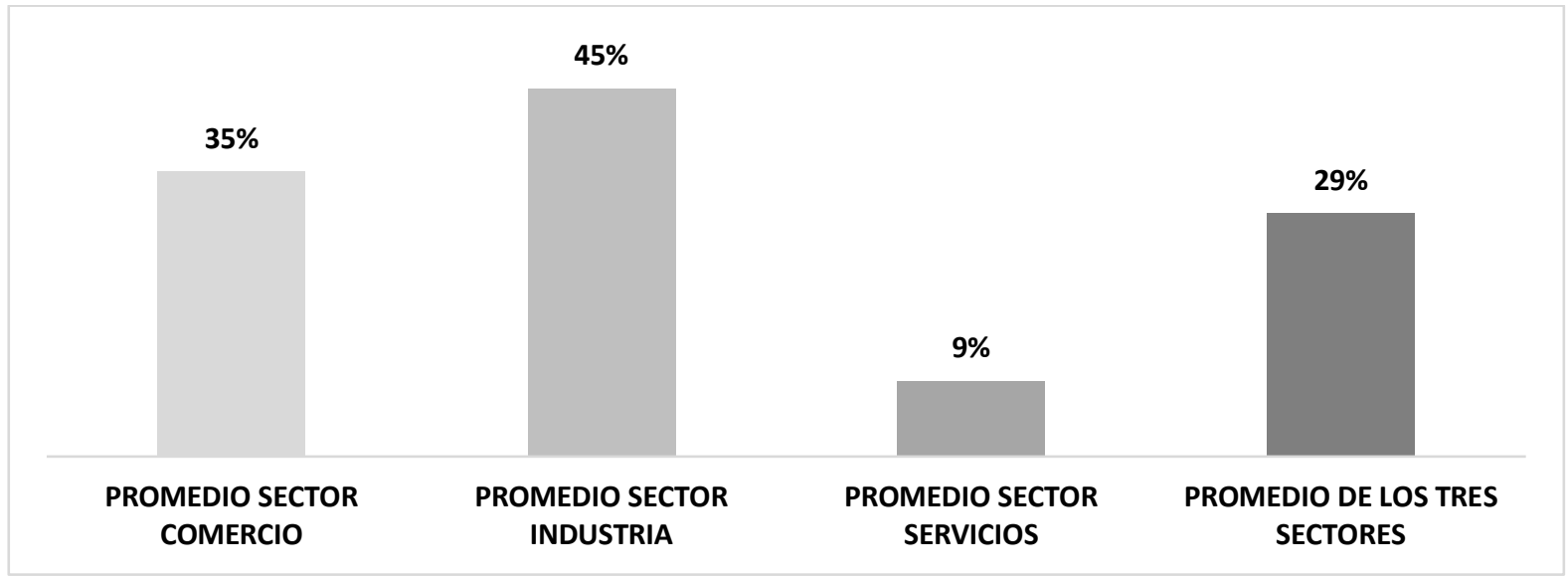

Figura 7: Variación porcentual promedio de los activos de una muestra de 12 empresas en el distrito de Los Olivos (2016 - 2017)

Fuente: Encuesta realizada a muestra de MYPE en Los Olivos - Elaboración propia

De manera general se observa un incremento promedio a nivel de los 3 sectores, comercio, industria, y servicios, de $29 \%$. A nivel sectorial, se observa un resultado positivo: comercio (35\%), industria (45\%) y servicios $(9 \%)$, resultados generados por la adquisición de nuevos equipos, maquinarias y mobiliarios, que buscan mayor productividad y mejores resultados a futuro.

Con respecto a si el Régimen MYPE Tributario ha tenido un impacto bastante significativo sobre el empleo generado por las micro y pequeñas empresas de los sectores, comercio, industria y servicios, del distrito de Los Olivos, la encuesta aplicada a una muestra de 12 microempresas de Los Olivos, arroja los siguientes resultados: un 92\% contestaron que sí han elevado su contratación de personal, y que incluso un 33\% señaló que han contratado más de 3 trabajadores, tal como se muestra en los siguientes gráficos. 


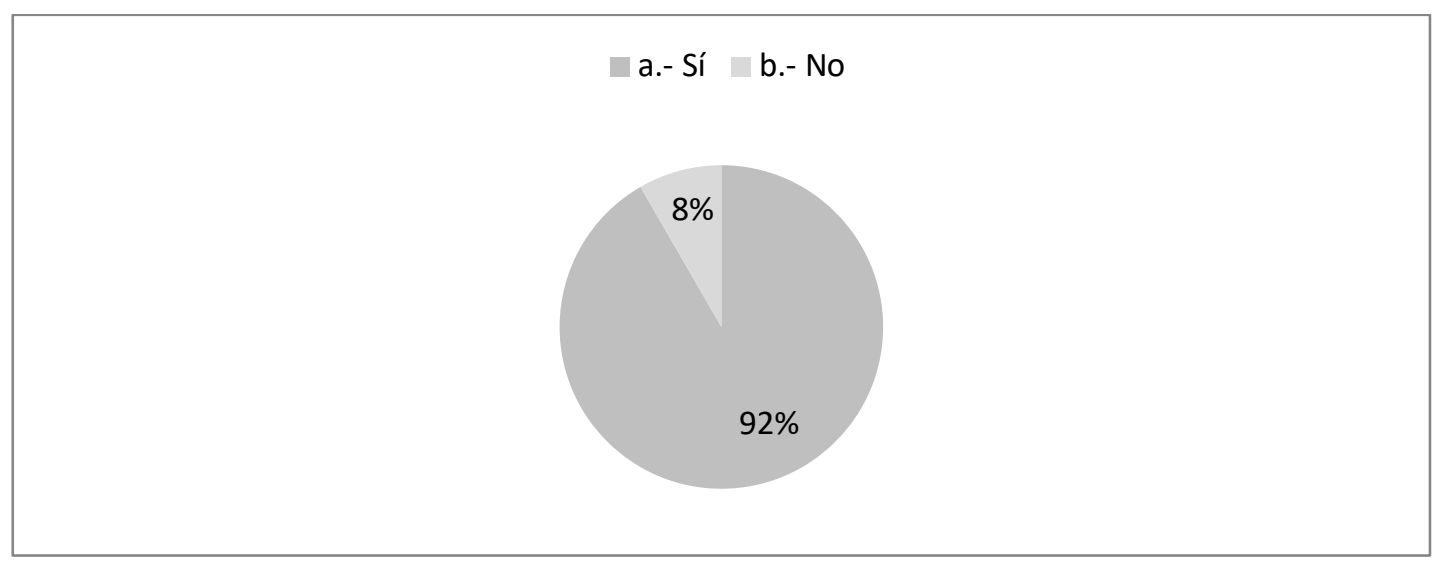

Figura 8: ¿Luego de acogerse al Régimen MYPE Tributario (RMT) elevó su contratación de personal?

Fuente: Encuesta realizada a muestra de MYPE en Los Olivos - Elaboración propia

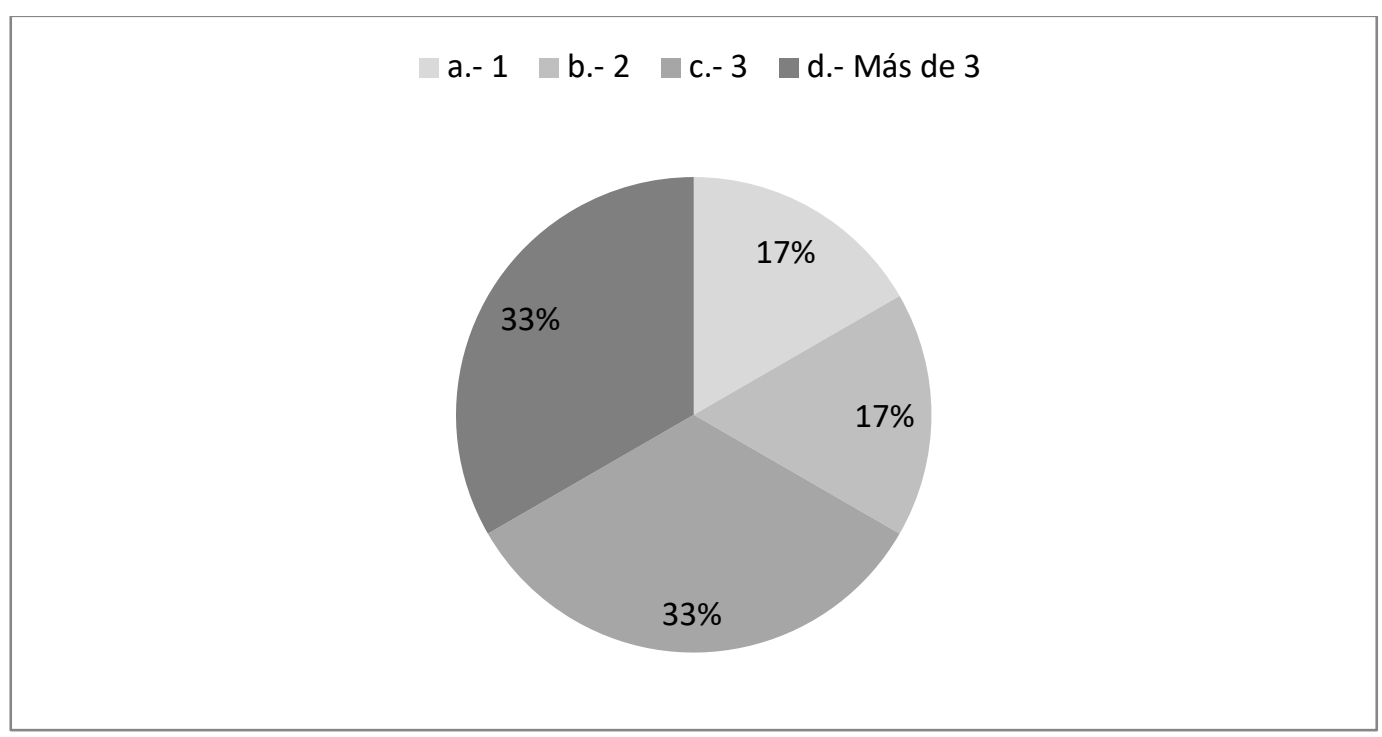

Figura 9: Luego de acogerse al Régimen MYPE Tributario (RMT) ¿cuántos trabajadores adicionales contrataron?

Fuente: Encuesta realizada a muestra de MYPE en Los Olivos - Elaboración propia 
Estos resultados pueden estar explicados por las decisiones tomadas por las empresas debido a que obtuvieron mayor liquidez generada por un menor pago a cuenta mensual y anual en el Régimen MYPE Tributario, el cual permitió ampliar sus actividades económicas y como consecuencia una mayor demanda de mano de obra.

De manera general entonces, se observa que se corrobora la hipótesis planteada, de que la Reforma del Impuesto a la Renta ha tenido un impacto medianamente significativo en el crecimiento de las micro y pequeñas empresas del distrito de Los Olivos, y su efecto ha sido de distinta magnitud en los sectores, comercio, industria y servicios.

\section{Discusión}

Del análisis de datos se puede concluir que el RMT a nivel de sectores económicos vemos que el impacto ha sido variado, como se puede apreciar en las figuras 2,3 y 4 , ello debido a que cada sector enfrenta diversos mercados, y el sector comercio siempre ha sido el más dinámico en la economía en su conjunto, mientras que el industrial y de servicios requieren de mayor tiempo de maduración de los negocios, en concordancia con los estudios realizados por Davidsson (1989) al indicar que las relaciones entre los resultados esperados son asimétricos al crecimiento.

Como consecuencia, se aprecia también la caída en las ventas y las utilidades, esto mostraría que de manera específica el impacto no ha sido favorable en el corto plazo para las MYPE, con la inclusión del RMT, situación contraria a lo que indica Bird y Wilkie (2012), cuando plantea que el efecto del impuesto debe generar un impacto positivo sobre el crecimiento. En tanto habría que observar en el transcurso de los años siguientes otros resultados, porque la investigación muestra que si hubo incremento de los activos fijos reflejados en inversión de equipamiento y mejora de infraestructura para las MYPE, lo que tiene efectos positivos sobre las ventas de la empresa en el mediano plazo.

Tal como se aprecia en la figura 5, el efecto variado que se observa en el pago del impuesto a la renta desde la vigencia del RMT, se debe a que cada sector tiene un comportamiento distinto, ello reflejaría que el sector servicios es más formal, mientras que en los sectores industria y comercio, existiría una mayor reticencia o reacción más lenta para el pago de impuestos, pudiendo afectar la recaudación del impuesto a mediano 
plazo, tal como lo verifico Macek (2014), y determino en su investigación que existe una relación negativa entre el crecimiento y el impuesto a la renta en reforma.

En virtud a lo indicado por Jacobs (2007), el incremento de contratación de puestos de trabajo muestra el impacto importante de la reforma tributaria sobre el crecimiento económico, ya que la nueva contratación de personal obedece a un mayor dinamismo de la producción, de perspectivas de mejora del negocio, tal como lo muestra la figura 8.

De los escenarios expuestos, se puede concluir que el régimen MYPE tributario para los empresarios, ha generado resultados distintos, no logrando evidenciar de forma contundente, la relación positiva a corto plazo entre la reforma del impuesto y el crecimiento de las MYPE en el distrito de Los Olivos. Pero si hay indicadores de inversión y contratación de mano obra, que deberían mostrar mejores resultados en el crecimiento en los próximos años.

\section{Recomendaciones}

Realizar estudios de investigación respecto al Regimen MYPE Tributario en cuanto a sus efectos en la formalización y crecimiento empresarial de las MYPE.

Recomendar a la Administración Tributaria que realice procesos de fiscalización a las empresas, en los aspectos de cumplimiento de los requisitos para acogerse al Régimen MYPE Tributario, e inspección y control de cumplimiento de sus obligaciones formales y sustanciales tributarias pues el Régimen MYPE Tributario a llevado a la atomización de empresas para conseguir menor pago de impuestos.

Sugerir a la Administración Tributaria, llevar a cabo actividades de promoción y publicidad del nuevo régimen tributario para que más micro y pequeños empresarios conozcan y puedan acceder a los beneficios que brinda.

Recomendar a la Dirección General de Política de Ingresos Públicos del Ministerio de Economía y Finanzas que realice una evaluación de los resultados y el desempeño de la recaudación por concepto del régimen MYPE tributario, y que se propongan mejoras a nivel administrativo para ser más eficaz en el cobro de impuestos. 
Exhortar al gobierno central, que participe con medidas de mayor apoyo comercial a las MYPE, a través de las compras estatales, que incluya procesos simples y menos burocráticos, que permita el aumento de sus ventas e impulse su crecimiento.

Sugerir al gobierno, a través del Ministerio de Economía y Finanzas, que elabore estudios económicos y que se estime el impacto en la recaudación, para proponer una nueva escala y alícuota intermedia entre el $10 \%$ y el 29.5\%, que permita seguir apoyando el crecimiento de la MYPE.

Evaluar los resultados económicos y financieros anuales a través de los estados financieros de las empresas acogidas al Régimen MYPE Tributario para identificar si están generando mayor productividad que les permita obtener mayores beneficios económicos y puedan consolidar su crecimiento, para conseguir esto, es importante que las empresas inviertan en elaborar sus estados financieros, para identificar sus resultados, que les permita tomar mejores decisiones, es conocido que por algunas limitaciones solo algunas empresas lo pueden elaborar.

Proponer al Ministerio de Economía y Finanzas que realice un estudio económico y que proyecte el impacto en la recaudación tributaria, para que se proponga una mayor tasa de depreciación tributaria para la deducción del gasto por la inversión de la MYPE en activos inmovilizados, con el objetivo de impulsar la adquisición de nuevas maquinarias y equipos que le permita mejorar su productividad.

Recomendar hacer otros estudios e investigaciones en el ámbito laboral-tributario, que permita relacionar el nuevo régimen tributario con la formalización laboral, para apreciar si el régimen MYPE Tributario contribuye con una mejora del empleo formal.

\section{Referencias bibliográficas}

Barbarán, N., Arteaga, P., Caballero, A., Rodríguez, W., Neciosup, E. (2018). Limitaciones que impiden el desarrollo empresarial de MYPES: distritos de Coishco, Chimbote, Nuevo Chimbote y Santa - 2017. (Informe de Investigación) Universidad San Pedro: Chimbote.

Bird, R., Wilkie, J. (2012). Designing Tax Policy: Constraints and Objectives in an Open Economy. International Center for Public Policy. 1 (1), 12-24. 
Davidsson, P.(1989). Entrepreneurship — and after? A study of growth willingness in small firms. Journal of Business. Venturing. 4 (1), 211-226.

Jacobs, B. (2007). Optimal Tax and Education Policies and Investments in Human Capital. 212-229. DOI: 10.1017/CBO9780511493416.014

Jones, L., Manuelli, R. (1999). On the Taxation of Human Capital. University of Minnesota - Department of Economics. 1 (1), 2-35.

Macek, R. (2014).The Impact of Taxation on Economic Growth: Case Study of OECD Countries. Review of Economic Perspectives 14(1), 309-328. DOI: https://doi.org/10.1515/revecp-2015-0002

Norregaard, J., Khan, T (2007) Tax Policy: Recent Trends and Coming Challenges. IMF Working Paper. Fiscal Affairs Department. 1 (1), 8-36.

Teixeira, A., Fortuna, N. (2003). Human Capital, Innovation Capablility and Economic Growth. Faculdade de Economia Universidade do Porto. 131 (1).

Zevallos, N. (2015) El Regimen Mype Tributario y el desarrollo empresarial en las empresas de transporte de carga en el distrito de huanuco-2017 (Tesis para obtener el grado de contador público) Universidad de Huánuco. Huánuco. 